\title{
EXPLORACIÓN DE LA DOMÓTICA EMOCIONAL: UNA PROPUESTA SENSORIAL EN LA RELACIÓN HOMBRE-ESPACIO
}

\author{
EXPLORATION OF EMOTIONAL DOMOTICS: \\ A SENSORY PROPOSAL IN THE MAN-SPACE \\ RELATIONSHIP
}

\section{CARLOS EDUARDO SILVA OSORES}

Centro de Innovación Tecnológica Fab lab ESAN. Universidad ESAN
La arquitectura refleja la intencionalidad del diseñador por transformar el espacio y contribuir a la percepción de confort del usuario. En este proceso integra tecnologías de la información y comunicación - domótica- intensificando su interacción con diversos elementos del entorno. Su aplicación ha permitido hasta ahora la comunicación unidireccional del usuario con el espacio, pero al incorporar el reconocimiento inteligente de emociones se transforma en bidireccional posibilitando la respuesta del espacio, adaptándose. El presente artículo explora este cambio radical en la relación usuario-espacio y propone un modelo conceptual basado en el confort como punto de partida para profundizar en su estudio.

domótica emocional, ambientes interiores inteligentes, relaciones humano-espaciales
NALDI SUSAN CARRIÓN PUELLES

Centro de Innovación Tecnológica Fab lab ESAN.

Universidad ESAN

Recibido: 7 de junio del 2019

Aprobado: 6 de agosto del 2019

doi: 10.26439/limaq2020.n006.4821

Architecture reflects designer's intentionality to transform space and contribute to user's comfort perception. Thus, it embraces information and communication technologies-domotics - to intensify its interaction with diverse enviromental elements. Hitherto, domotics implementation has allowed unidirectional communication from users to space. However, when incorporating intelligent emotion recognition systems, communications becomes bidirectional, enabling an adaptative response from space. This article explores such radical change within userspace relationship and proposes a conceptual model based on comfort as a starting point for further study.

emotional domotics, smart indoor environments, human-space relationships 


\section{INTRODUCCIÓN}

Bajo una mirada fenomenológica, el espacio refleja la sociedad que lo habita, encontrándose, entonces, su forma condicionada a la intencionalidad del hombre (Bollnow y D'Ors, 1969). De esta manera, tal como indica Stanley (2015), la arquitectura, recoge las dinámicas sociales y valores culturales reflejando a la sociedad, y se convierte en un registro de ese proceso colectivo para la posteridad. Así, a partir del estudio arquitectónico, se explica, por ejemplo, cómo la alta valoración del sentimiento de seguridad e intimidad en la cultura china se ve expresada en un diseño arquitectónico ajustado a las necesidades del ser humano, fiel reflejo de la noción extendida de practicidad sin los usuales sobredimensionamientos de la cultura occidental (Stanley Okwudili, 2015). Sin embargo, la arquitectura también influye en el comportamiento de sus ocupantes tanto a nivel individual, transmitiendo un mensaje a través del diseño de interiores, como a nivel grupal, guiando las interacciones sociales que se ven reflejadas en las pautas del diseño de planta y que están alineadas con el propósito de la edificación (Barsky y Nash, 2002; Ryu y Jang, 2007; Stanley Okwudili, 2015). Sin embargo, actualmente observamos cambios mayores en el paradigma hombre-espacio, en el sector edificación; con la inclusión de las TIC se ha logrado establecer nuevas formas de relación antes no registradas (Aiello M., Marchese M., Busetta P, y Calabrese G., 2004). A partir de esta inserción, nace la rama de la domótica emocional, en la búsqueda por conectar el control de los espacios cerrados a las emociones de sus ocupantes o usuarios (Navarro-Tuch et al., 2016).

En este contexto, el propósito del presente artículo es explorar la apropiación, desde el lado de la arquitectura, de esta rama altamente estudiada desde el sector de las TIC. Se busca, así, enlazar la domótica emocional y conocer su influencia potencial en la relación hombre-espacio relacionando tres conceptos: confort, tecnología y espacios adaptables. De este modo, en la revisión de la literatura se establece el concepto de confort y se explica la relación que guarda con el diseño arquitectónico. Posteriormente, se define el alcance de los ambientes inteligentes describiéndose el modelo general para su diseño y se introduce la rama de la domótica emocional. Seguidamente, se describe el método empleado para el levantamiento de los datos exploratorios y sus resultados. Finalmente, se discuten los hallazgos y se presentan las conclusiones.

\section{REVISIÓN DE LA LITERATURA}

\section{Confort}

El confort es un concepto complejo afectado por factores tanto externos como internos de cada individuo y puede ser definido como la existencia de todas las condiciones necesarias dentro de un espacio determinado disminuyendo la probabilidad de que una persona prefiera trasladarse para mejorar su estado (Bujdei y Moraru S. A., 2011). Así, se pueden establecer tipologías de confort de acuerdo a los siguientes parámetros: térmico, visual, acústico, calidad de 
aire, estabilidad (movimientos indeseados, vibraciones), seguridad, factores económicos y costumbres horarias (periodicidad de actividades / fenómenos) (Bujdei y Moraru, 2011). A continuación, definimos algunas de los tipos de confort:

- Térmico: hace referencia al estado mental que expresa satisfacción con las condiciones térmicas del ambiente, facilitando la realización de actividades en óptimas condiciones (Al Horr et al., 2016). Depende de factores físicos, como la temperatura y velocidad del aire; de factores orgánicos, como son la edad, sexo y nacionalidad de usuario y, finalmente, de factores subjetivos relacionados con el individuo, como son el tipo de ropa que utiliza, actividades que desempeña, condición social, entre otros (Bujdei y Moraru, 2011). Así, resulta la tipología más fácil de definir existiendo estándares para su medición (Al Horr et al., 2016).

- Acústico: se relaciona directamente con la capacidad de un lugar de proteger a los ocupantes del ruido y ofrecer un ambiente acústico según las tareas desarrolladas (Al Horr et al., 2016). Este aspecto puede incidir sobre el desempeño de los habitantes del espacio, llegando a generar estrés, desconcentración, reducción del desempeño operativo, deterioro de la memoria e incremento del ritmo cardíaco (Bujdei y Moraru, 2011).

- Visual: impacta en el bienestar y la productividad en espacios cerrados y está relacionado con la iluminación del espacio, el uso de color e inclusive con la percepción de la densidad dentro de una habitación. Estas características son factores que afectan la productividad y el estado psicológico de los ocupantes (Al Horr et al., 2016).

El confort, independientemente de su tipología, se compone de factores objetivos y subjetivos, siendo este último la razón de la dificultad para cuantificar el resultado (Bujdei y Moraru, 2011). La investigación realizada por Fransson (2007) determinó que los factores subjetivos primaban por encima de los objetivos. Por ejemplo, en el caso del confort termal, la ropa utilizada en cierto momento tiene mayor significación en la percepción de comodidad que la temperatura, velocidad del aire o humedad que se experimentan, estableciéndose que la percepción del confort, independientemente de su tipología, se ve influenciada en mayor medida por factores intrínsecos a las personales (Fransson, Västfäll, y Skoog, 2007).

En cuanto a la influencia de la tecnología sobre el confort, se ha comprobado que su implementación en espacios interiores genera un incremento en la autonomía de los ocupantes incidiendo profundamente en los niveles de confort que experimentan (De Carvalho Correia, De Miranda, y Hornung, 2013). Así, por ejemplo, la investigación liderada por Ryu y Jang (2008) al analizar la relación entre los factores ambientales en restaurantes y la intención de comportamiento del consumidor, establecieron que la ambientación (música, temperatura y olor) resulta determinante en la experiencia de los comensales para su fidelización. Mientras que, a través del trabajo desarrollado por Barsky 
y Nash (2002) se identificaron las emociones clave generadas en los clientes potenciales, asociadas a cada característica ofrecida por la diversa gama de la oferta hotelera. Como en el caso de los hoteles de lujo, donde los huéspedes esperan en un espacio visualmente lujoso y relajante, con una atención superior al estándar, pudiéndose optar por mejorar dichas características e influir en su fidelización (Barsky y Nash, 2002). Sin embargo, es preciso reconocer que la relación entre la calidad de los factores interiores de un espacio y el bienestar de sus ocupantes, es compleja y difícil de establecer, pudiendo tener efectos a corto y largo plazo (Al Horr et al., 2016).

Cabe preguntarse entonces si la incorporación de sensores que capten data objetiva y medible es suficiente para garantizar la generación de ambientes placenteros o siquiera ambientes neutros, cuando realmente se desconocen otras variables de la ecuación. ¿Cuál es la implicancia de la mayor influencia de los factores subjetivos sobre la comodidad de los ocupantes? Por ejemplo, en un escenario donde se persigue brindar confort térmico, ¿se puede confiar en que el control de la temperatura, humedad y velocidad de aire es suficiente? o, por el contrario, ¿está la percepción del ocupante, sujeta, finalmente, a factores internos que desconocemos de manera directa, y en los que debemos enfocarnos? ¿Cómo podemos finalmente garantizar el confort si no tenemos acceso a toda la información? ¿Puede la incorporación de tecnología aproximarnos en la medición de estos factores subjetivos?

\section{Ambientes inteligentes}

Dentro del desarrollo de las TIC, los sistemas inteligentes incorporan la capacidad de interpretar acciones y gestos humanos y las traduce a necesidades a las cuales responder; de esta forma, se puede inferir que cuando estos sistemas se conjugan con infraestructura, dan paso a lo que se conoce como "ambientes inteligentes" (De Carvalho Correia et al., 2013; Ekman, Paul y Keltner, Dacher, 1970; El Murabet y Abtoy, 2019; Fernández-Caballero et al., 2016; Fransson et al., 2007; Navarro-Tuch et al., 2018). De acuerdo a lo analizado por El Murabet y Abtoy (2019), no existe actualmente un consenso en cuanto a su definición, encontrándose un uso alterno e indiferenciado del término "domótica”. Así, este término es conceptualizado también como el campo, la rama de la arquitectura que emplea las tecnologías de la información y comunicación junto con sistemas de ingeniería para brindar el soporte necesario a los ocupantes de un determinado espacio permitiéndoles mantener y realizar sus actividades diarias y proseguir con sus actividades sociales (El Murabet y Abtoy, 2019). Estos espacios recogen información de las preferencias y comportamiento del usuario, la procesan y aprenden, tomando decisiones con base en lo aprendido y entendiendo las necesidades de los ocupantes (Montalbana, Garzo y León, 2009).

En la parte práctica existen distintas plataformas y planteamientos para la implementación y manejo de ambientes asistidos en viviendas, que buscan incorporar y mantener atributos básicos de interoperabilidad, escalabilidad, confidencialidad y seguridad (El Murabet y Abtoy, 2019). Por el lado académico se ha estudiado el enlace entre los ambientes inteligentes y el confort 
(Bujdei y Moraru, 2011; Fernández-Caballero et al., 2016; Fich et al., 2014; Montalbana et al., 2009; Navarro-Tuch et al., 2018; Valencia, 2019), contándose con varias investigaciones aplicadas al sector salud (Fernández-Caballero et al., 2016; Fich et al., 2014; Montalbana et al., 2009; Navarro-Tuch et al., 2018; Valencia, 2019), al estudio del manejo del confort en oficinas (Bujdei y Moraru, 2011), proponiéndose estudiar su aplicación en sitios donde se pretenda controlar las condiciones interiores para influenciar el comportamiento de individuos (El Murabet y Abtoy, 2019). A pesar de lo hasta ahora aplicado e investigado, existe en ambas áreas, la práctica y la académica, una falta de estandarización respecto de los factores que se deben tener en cuenta para el desarrollo de dichos sistemas (El Murabet y Abtoy, 2019). En ese sentido, De Carvalho (2013) presenta un marco conceptual que establece una triada entre el usuario, interacción por gestos y domótica considerando seis dimensiones cruciales:

- Accesibilidad: desde el punto de vista tecnológico se percibe como el atributo de calidad de los sistemas de software y hardware. Viendo la tecnología como un facilitador para la interacción gestual, los atributos que debe presentar la tecnología se enmarcan en el nivel de comprensión, operabilidad, percepción de complejidad, y compatibilidad con otras tecnologías que la convierten en más accesible para la diversidad de usuarios con los que se cuenta.

- Usabilidad: se encuentra ligada a la facilidad con la que los usuarios pueden manejar la tecnología. Los dispositivos que poseen interfaces gráficas ayudan a los usuarios a reconocer comandas en vez de obligarlos a recordar. En el caso de sistemas de reconocimiento de gestos, es importante considerar la ergonomía del movimiento que no se logra con las tecnologías wearable. Otros principios para considerar en la usabilidad son la consistencia, confiabilidad y escalabilidad de la solución.

- Personalización: desde el punto de vista tecnológico se percibe como el atributo de que permite adecuar la tecnología a los requerimientos del usuario. Sin embargo, analizando el registro gestual, dos personas nunca realizarán un mismo gesto de la misma forma y bajo esa premisa, el diseño de los sistemas de reconocimiento debe ser robusto y flexible permitiendo capturar la complejidad de las diferencias entre las personas y sus gestos, y ampliar su "vocabulario".

- Ambigüedad: la falta de claridad en los movimientos gestuales dificulta su determinación produciendo ambigüedad. Este grado de complejidad puede aumentar teniendo en cuenta que un mismo gesto puede tener distintos significados y que dicha complejidad puede verse exacerbada por el contexto y la percepción del receptor que lo decodifica. Incluso un mayor grado de complejidad se produce cuando en un mismo contexto se presenta el síndrome de inmersión donde la comunicación entre hombre-robot y hombre-hombre se ve comprometida al confundir quién es el receptor del mensaje. 
Figura 1

Marco concep-

tual de aspectos sociotécnicos

de interacción

gestual

Fuente: Carvalho

Correia et al.,

2013, p. 308
- Antropología: considera la capacidad del uso generalizado de tecnologías de reconocimiento gestual teniendo en consideración que un solo gesto puede tener una gran variedad de significados en dos localidades distintas, reconociéndose en este hecho el factor antropológico.

- Privacidad: en referencia a este aspecto en el acceso y manejo de los datos que se registran y analizan; está ligada al lugar donde está implementada la solución pudiendo afectar el tipo de relaciones.

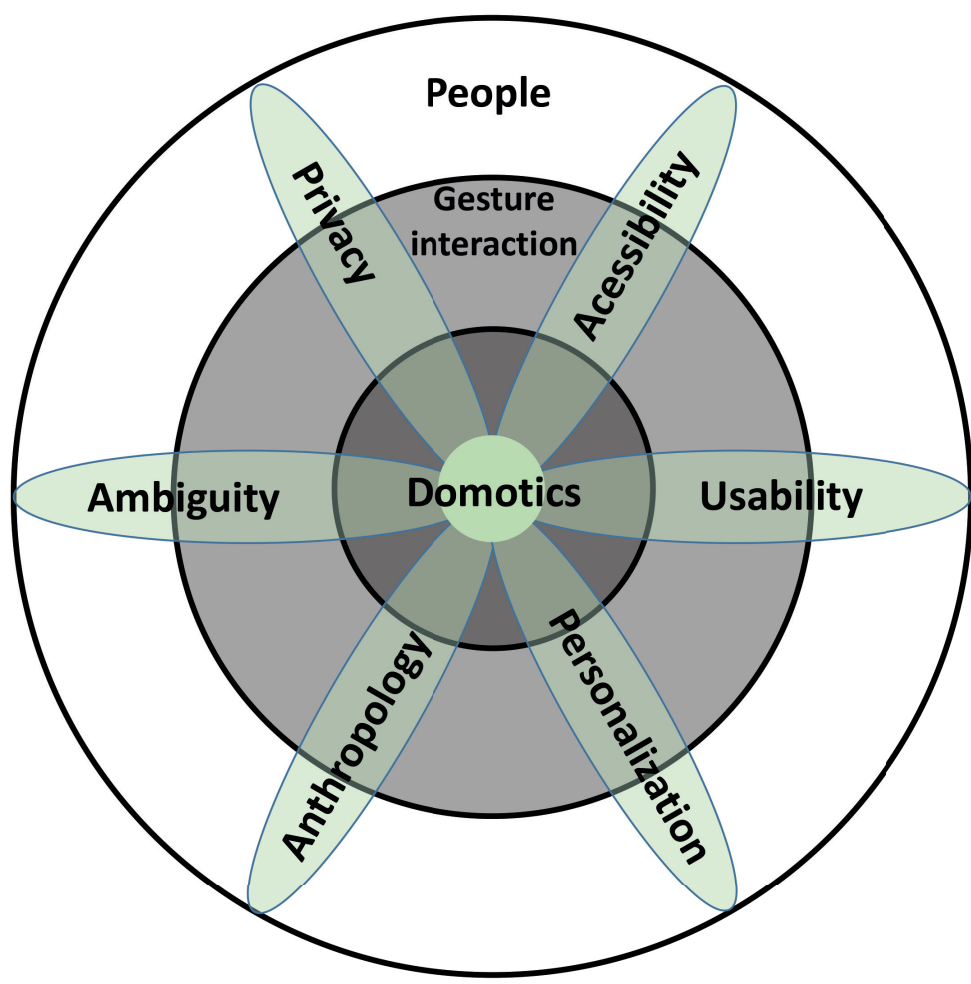

\section{Domótica emocional}

Los ambientes inteligentes, en la búsqueda por mejorar la experiencia del ocupante, son capaces de obtener data y procesarla dinámicamente para generar conocimiento sobre el entorno y sus ocupantes; asimismo, facilita que con determinadas acciones se puedan modificar ciertas condiciones del espacio (Barsky y Nash, 2002; Ryu y Jang, 2007; Stanley Okwudili, 2015). Cuando se busca que la comunicación entre hombre y máquina sea lo más intuitiva y fácil posible, según lo explorado por De Carvalho, se incorpora el reconocimiento de gestos, al ser una forma de comunicación natural para el hombre. Sin embargo, se pone de relieve la limitación de solo basarnos en 
gestos, siendo estas construcciones socioculturales que varían de acuerdo a diversos factores. Por ello, al hablar de una "interacción natural de gestos" se hace en realidad referencia al registro y utilización de movimientos simples y fáciles de aprender (de Carvalho et al., 2013).

Iraitz Montalbana (2009) promueve como una oportunidad en la mejora de la capacidad de respuesta de los ambientes inteligentes, la incorporación de la estimación probabilística de emociones, entenderlas detrás del comportamiento. Esta intersección entre los ambientes inteligentes y el reconocimiento de emociones ha dado origen a la domótica emocional, que tiene como objeto el desarrollo de sistemas de control no intrusivos de espacios cerrados manejados a partir de los estados emocionales de sus ocupantes y la búsqueda por elevar el nivel de confort (Navarro-Tuch et al., 2016). Al respecto, se ha visto facilitada la creación de códigos faciales para el reconocimiento particular de estado de ánimo (Ekman y Keltner, 1970; Navarro-Tuch et al., 2016), basados en la factibilidad de identificar un conjunto de emociones básicas cuya expresión facial es transversal e independiente de la cultura del individuo, siendo seis de ellas (felicidad, tristeza, furia, miedo, sorpresa y disgusto) estudiadas de manera recurrente en diversos contextos culturales (Ekman y Keltner, 1970).

En este contexto, Fernández-Caballero (2016) propone la configuración de la domótica emocional aplicada al sector salud, pero cuya base puede extrapolarse a otras situaciones y plantea, a través de un trabajo experimental, una composición de seis procesos que deben llevarse a cabo para el control de los ambientes buscando la regulación de las emociones:

- Detección facial de emociones: identifica la emoción base del usuario.

- Detección de comportamiento: identifica el comportamiento de la persona.

- Detección de excitación y placer: identifica el nivel de excitación (intensidad de la emoción) y de placer del paciente.

- Fusión de resultados: pondera la estimación del estado anímico del sujeto en estudio con base en la lectura de los "detectores" "utilizados, elevando la probabilidad de eficiencia en la detección de la emoción.

- Toma de decisión: a partir del resultado se establecerá el estado anímico y emocional objetivo, al cual se busca llevar al sujeto de estudio.

- Actuador: proceso encargado de activar los estímulos en el ambiente para modificar la emoción y comportamiento.

1 Los detectores son un conjunto de sensores y algoritmos que recolectan y procesan datos específicos para estimar la probabilidad de estados de interés. 
Figura 2 .

Arquitectura de

domótica emo-

cional

Fuente:

Fernández-

Caballero et al.,

2016, p. 57

Fernández-Caballero et al. (2016) proponen la identificación del estado del individuo estudiado con base en el modelo circunflejo de afecto ${ }^{2}$ de Russel (1980), conceptualizando una extensión del modelo (ver figura 3) representando emociones interconectadas entre ellas y relacionadas con el nivel de excitación (eje vertical) y nivel de placer (eje horizontal) (Fernández-Caballero et al., 2016). Los procesos de detección de la configuración planteada estiman el estado emocional para ubicarlo en algún punto del círculo de conexión de las emociones; así, el proceso de toma de decisiones predice el mejor camino para llevar al sujeto a un estado de placer con excitación neutral (FernándezCaballero et al., 2016).



2 El modelo circunflejo de afecto presenta gráficamente la interrelación entre las emociones, nivel de placer y excitación en un círculo. 




Figura 3.

Modelo circunfle-

jo de afecto

Fuente:

Fernández-

Caballero et al., 2016, p. 59

Los autores anteriormente citados detallan que, en el caso de la selección de opciones durante el proceso de toma de decisiones, existen estudios que experimentan sobre la modificación de factores físico-ambientales. Por ejemplo, se han utilizado como reguladores de emociones dos parámetros: la música, que induce a estados neutrales al controlar el tempo y ritmo; y el color de luz, que puede inducir a los pacientes a un estado de inactividad placentera. Así, se presenta la propuesta de incluir una capa de análisis de factores físicos que tiene por objeto predecir el estado emocional resultante (Fernández-Caballero et al., 2016). 




Figura 4. I METODOLOGÍA

Modelo circunfle-

jo para regulación

de emoción

usando música

Fuente:

Fernández-

Caballero et al.,

2016, p. 67
Debido a la naturaleza exploratoria del presente artículo y la cantidad incipiente de información académica, consideramos aplicar los dos primeros pasos de la metodología propuesta por Eisenhardt (1989):

1. Inicialmente se establece la pregunta de investigación: ¿Hasta qué punto la domótica emocional ha impactado en la relación hombre-espacio desde la perspectiva de la arquitectura?

2. Posteriormente se realiza el levantamiento de información en el motor de búsqueda especializado Springer Link cubriendo el período 2001-2019 para cuantificar el número de textos académicos relevantes según las palabras clave smart indoor environment y emotional domotics.

\section{RESULTADOS}

La búsqueda de la frase smart indoor environment en el SpringerLink arrojó 9593 resultados en distintas disciplinas, siendo las más predominantes en ciencias de la computación e ingeniería (tabla 1). Se recalca que si bien se localizaron registros en la disciplina de arquitectura / diseño, los artículos encontrados estudian las áreas de teoría e historia de la arquitectura, diseño, urbanismo y ciudades. La revisión no arrojó artículos científicos, textos de conferencias y libros que se relacionen al estudio del impacto de la tecnología en la relación hombre-espacio. 


\begin{tabular}{lc}
\hline Disciplina & Cantidad \\
\hline Computer Science & 3553 \\
Engineering & 3474 \\
Environment & 313 \\
Energy & 289 \\
Medicine y Public Health & 270 \\
Architecture / Design & 23 \\
Others & 1671 \\
Total & 9593 \\
\hline
\end{tabular}

Tabla 1.

Búsqueda

de la frase

smart indoor

environment en

SpringerLink

Elaboración

propia

Tabla 2

Búsqueda de emotional domotics en SpringerLink

Elaboración propia 
Tabla 3

Búsqueda de

frase smart

indoor environ-

ment emotional

domotics en

SpringerLink

Elaboración

propia

\begin{tabular}{lc}
\hline Disciplina & Cantidad \\
\hline Engineering & 3 \\
Medicine y Public health & 1 \\
Philosophy & 1 \\
Social sciences & 1 \\
Total & 6 \\
\hline
\end{tabular}

\section{DISCUSIÓN}

\section{Smart environments versus emotional domotics}

Nos toca reflexionar aquí sobre los conceptos de "ambientes inteligentes" y "domótica" en los que se basa nuestra búsqueda de literatura, no siendo nuestra intención crear un debate en cuanto al término que debe emplearse, por el contrario, adoptamos lo referido en el "ciclo de vida de los constructos académicos" propuesto por Hirsch y Levin (1999), reconociendo que la definición, tipologías y determinantes se seguirán construyendo de manera progresiva con el avance de las investigaciones académicas. De esta manera, esta exploración busca agregar una perspectiva de evaluación que contribuya a la discusión actual e incentive a otros investigadores a experimentar y aportar en la construcción de una mejor definición del concepto, modelo y teorías conexas.

En este contexto, el análisis de la revisión bibliográfica develada por ambas conceptualizaciones revela que en los últimos 20 años el $73 \%$ de los artículos relacionados con el término smart indoor environment está directamente relacionado con temas tecnológicos (ingeniería y ciencias de la computación). La revisión de los pocos textos encontrados en la disciplina de arquitectura, que representan menos del $0,3 \%$ del total, no guardan relación con aplicaciones tecnológicas específicas, sino más bien con aquellas relacionadas al urbanismo o casos de estudios sociales. Cuando se repite el procedimiento utilizando los términos emotional domotics y smart indoor environment emotional domotics los resultados disminuyen drásticamente y se desligan de la arquitectura. Ello evidencia la brecha en el estudio de la domótica emocional y su efecto en los ambientes, desde una perspectiva arquitectónica, y abre las posibilidades de estudio en el campo. 


\section{Revisión de literatura}



\section{Revisión de literatura}

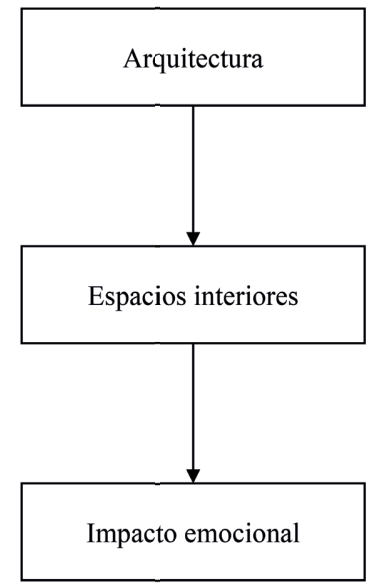

\section{La interacción con la arquitectura}

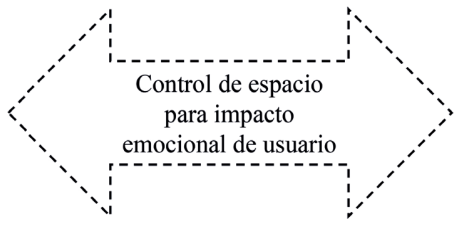

Figura 5.

Identificación de brechas en la literatura del diseñador en la transformación espacial; apreciamos que el arquitecto, al diseñar una estructura, plasma una intencionalidad que ve reflejada en distintos aspectos. Por un lado, la distribución de espacios promueve interacciones sociales; por otro, acabados como el color e intensidad de luz y control térmico pueden afectar el comportamiento individual. Así, la relación hombre-espacio tiene como objeto final la persuasión del ocupante para la realización de actividades con actitudes determinadas. Bajo esta perspectiva, se establece una relación unidireccional compuesta por tres elementos: diseñador-espacio-ocupante.

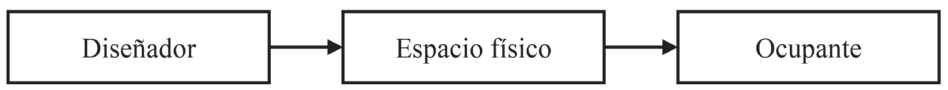

En esta correspondencia, cualquier modificación a la intencionalidad subyacente del espacio tiene que ser realizada por un agente externo al espacio mismo y que toma la posición de diseñador. De esa forma, la arquitectura se convierte en un medio disuasorio pasivo, sin capacidad de cambio y dependiente del propósito original ¿Qué sucede cuando ese agente externo se embebe en la arquitectura? El desarrollo tecnológico actual nos permite analizar ese contexto. Cuando se agregan elementos digitales capaces de medir y controlar la ambientación respecto de la infraestructura, el medio disuasorio pasivo se convierte en un medio regulador. Así, los sistemas inteligentes aplicados en la domótica emocional asumen ese rol transformador sobre la infraestructura. Al ser capaces de interpretar las emociones y decidir cómo modificar la ambientación en la búsqueda de mejorar el confort e incidir sobre

\section{Elaboración}

propia

\section{Figura 6.}

Relación

hombre-espacio tradicional

Elaboración

propia 
Figura 7

Relación

hombre-espacio con tecnología

Elaboración propia el humor de sus ocupantes, otorgan la capacidad de adaptabilidad afianzando el rol regulador del espacio, adicional al propósito inicial del diseñador.

Considerando lo expuesto, es posible esbozar correspondencias bidireccionales en la relación espacio-hombre siendo la tecnología un facilitador de la detección y regulación de las emociones del individuo (figura 6). Así, las relaciones se establecen entre cuatro elementos base: el diseñador, quien establece un propósito inicial al sistema; el ocupante, quien usa el espacio para actividades determinadas; el espacio físico/digital, que es un entorno físico al que se le han instalado sensores para captar el estado del ocupante y los actuadores (y así controlar las condiciones físicas; y el sistema inteligente, que estima el estado del usuario y decide cómo modificar el ambiente.

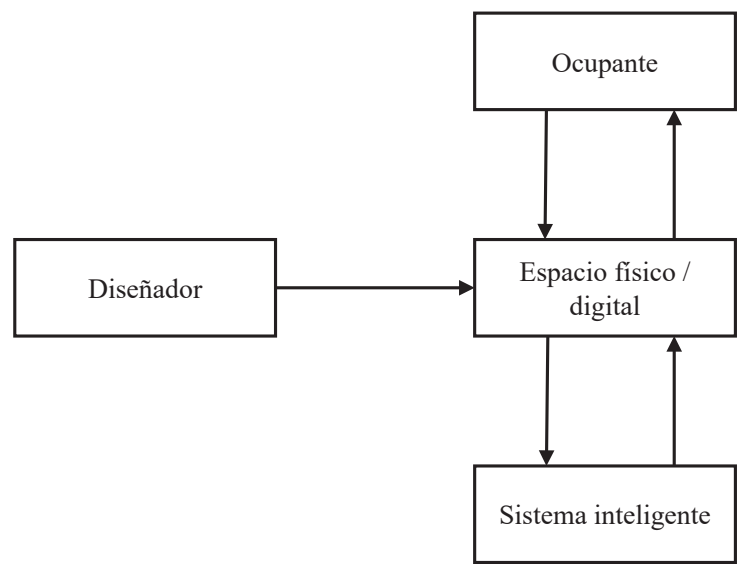

La conjugación del espacio físico y digital, del sistema inteligente y ocupante permite modificar la intencionalidad del espacio y reflejarla a partir de cambios ambientales determinados sin requerir al diseñador. Por ejemplo, un salón que tenga instalado un sistema de calefacción controlado por computador y sensores puede analizar al ocupante para decidir si el espacio requiere enfriarse o mantener el calor, tomando como base el comportamiento del sujeto y obviando las condiciones ambientales externas. De esta forma, el entorno deja su rol como un medio de intencionalidad y se convierte en el gestor de sus propios cambios según las necesidades.

Tomando como base la relación hombre-espacio de la figura 6, se propone que el flujo de intencionalidad para la regulación autónoma en la domótica emocional se centre en dos elementos: el ocupante y el sistema inteligente. Inicialmente, el cuerpo humano procesa las condiciones del ambiente y en caso de incomodidad dará una respuesta emocional y conductual según el contexto. En esta primera etapa, la información recolectada por los sentidos es interpretada por la psique produciendo una respuesta conductual correlacionada con el grado de confort del individuo. De esta forma, el cuerpo puede 
expresar su conformidad o deseo de cambio relacionada con la emoción que experimenta. En una segunda fase, el sistema inteligente recoge la información conductual, la interpreta y decide su necesidad de intervención, pudiendo seleccionar qué parámetros modificar en el ambiente. En esta etapa, la información conductual es captada por los sensores del entorno y convertida en información digital; el sistema inteligente recolecta el interés del hombre y propone, de ser el caso, un conjunto de condiciones con la intención de mejorar el estado actual. Finalmente, el sistema inteligente ordena al espacio físico/digital y altera determinadas condiciones que serán reconocidas por los sentidos. En esta última etapa, la información digital se convierte en estímulo generando una nueva emoción que volverá a alimentar el sistema.

De lo anterior se desprende que el espacio físico/digital se vuelve un intermediario entre la intencionalidad del ocupante (que solicita una acción) y el espacio-sistema inteligente (que busca cumplir con la solicitud remitida). Sin embargo, gran parte de la literatura revisada se centra en el análisis del sistema inteligente desde una perspectiva tecnológica, dejando de lado su interacción con la dimensión humana y espacial. Existe, por lo tanto, una oportunidad de ampliar el estudio sobre las correspondencias bidireccionales entre el espacio físico/digital y el ocupante desde una mirada arquitectónica.

Si tomamos como referencia el modelo presentado por Carvalho Correia (2013) y la propuesta de arquitectura de Fernández-Caballero (FernándezCaballero et al., 2016), la domótica emocional aporta solidez a las dimensiones de usabilidad y personalización de manera directa. El sistema reacciona ante la muestra de incomodidad y puede conducir al ocupante a un estado optimizado según las necesidades y características individuales. Asimismo, al basarse en la lectura de emociones primarias y universales, se reduce el inconveniente de la dimensión antropológica, puesto que se minimiza la influencia del contexto cultural.

Siguiendo con el análisis, la dimensión de privacidad debe ser analizada desde dos enfoques complementarios. Considerando la experiencia de usuario, se debe minimizar la percepción de intrusión en actividades de índole privada para evitar la posibilidad de generar incomodidad. Desde una mirada técnica, es importante minimizar la vulnerabilidad del sistema inteligente y evitar el robo de información personal y/o al menos, en el peor de los escenarios, permitir la intervención remota sobre el recinto controlado.

Habiendo establecido la nueva relación hombre-espacio se pueden analizar los factores determinantes, que, al ser alterados, incidirán sobre los elementos del modelo, existiendo, además, una interrelación entre los tres factores determinantes:

- Nivel tecnológico: los sistemas inteligentes podrán tomar mejores decisiones conforme evolucionen las capacidades tecnológicas y los modelos predictivos de comportamiento y reconocimiento de emociones. Dentro de este ítem se incluye el desarrollo de mejores sensores. 
Figura 8

Relación entre el

nivel de

tecnología y el de adaptabilidad

Elaboración

propia
- Nivel de adaptabilidad: las viviendas y oficinas cuentan con dispositivos que pueden alterar el espacio colocadas según las consideraciones iniciales del diseño arquitectónico, sin tener previsto actuar en respuesta a emociones específicas: apagado automático de luz, aire acondicionado, persianas automáticas.

- Nivel de confort: hace referencia al grado de comodidad que los ocupantes perciben en un espacio debido a las condiciones.

\section{Relación tecnología-adaptabilidad}

La adaptabilidad de los espacios está sujeta al desarrollo tecnológico de dispositivos de control ambiental. Si bien hoy en día no es difícil encontrar equipos que controlen música, intensidad, color de luz y temperatura, sería interesante ver el impacto que tendrían máquinas que puedan alterar fácilmente la distribución de espacios y olores o artículos de uso interior (como mesas, sillas, repisas, etcétera) que sean modulables.



\section{Relación adaptabilidad-confort}

Con la inclusión de equipos que modifiquen el espacio es posible cubrir nuevas clasificaciones de confort que actualmente no se consideran, contando con mayor cantidad de canales para regular el estado emocional. Ello puede ayudar a tener un mejor manejo emocional del ocupante. 


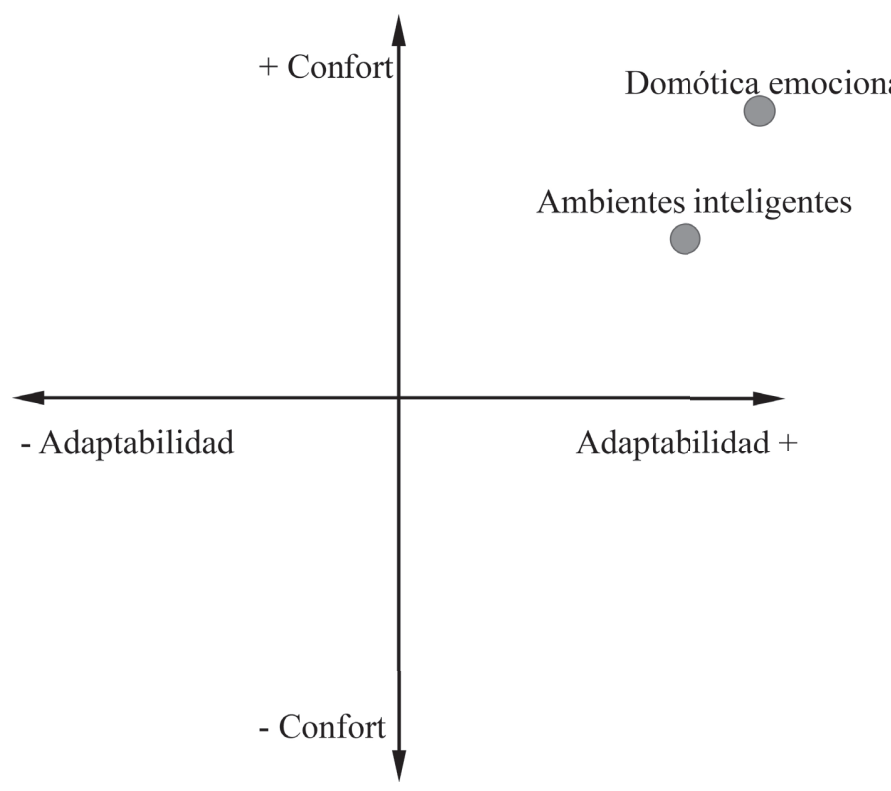

Figura 9 .

Relación entre

el nivel de adap-

tabilidad y el de

confort

Elaboración

propia

\section{Relación tecnología-confort}

El desarrollo de sistemas inteligentes permite la regulación automática del espacio para lograr el confort del ocupante. Es importante considerar que los dispositivos de control ambiental por sí solos no automatizan la respuesta, sino que requieren de un cerebro virtual que estime y tome decisiones.

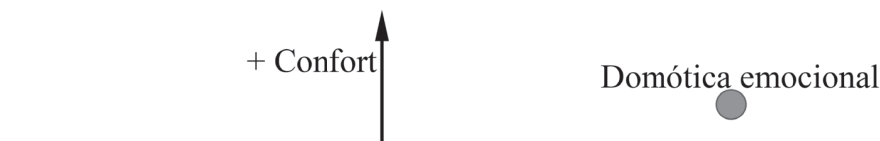

Figura 10.

Relación entre

el nivel de tecnología y el de Ambientes inteligentes confort

Elaboración propia

- Confort 
Figura 11

Relación entre

los niveles de

tecnología,

adaptabilidad y

confort

Elaboración

propia

\section{Relación tecnología-adaptabilidad-confort}

Visto integralmente, el nivel tecnológico es el elemento catalizador que aporta tanto al nivel de adaptabilidad espacial como al de confort. Su incorporación en el modelo refuerza la relación adaptabilidad-confort ya que, al incorporar sensores y actuadores en el espacio, permite entender mejor el entorno real y a quienes lo habitan.



\section{CONCLUSIONES}

La revisión bibliográfica reveló que la mayoría de investigaciones realizadas sobre domótica emocional se realizan bajo perspectivas ingenieriles, dejando el terreno abierto para considerar enfoques arquitectónicos. Ello conlleva a distanciarse de los aspectos técnicos de la tecnología y a profundizar en el estudio de la relación que el hombre entabla con su entorno. Al explorar esta ruta se propone una interrelación entre cuatro elementos: diseñador, ocupante, espacio físico/digital y sistema inteligente. Sometido a un análisis longitudinal, la conceptualización del diseño arquitectónico inicial no puede ser modificada, sin embargo, podemos incrementar o inhibir ciertos atributos con base en modificaciones del espacio o ambientación interior conjugando variaciones sobre los otros tres elementos. El ocupante, el espacio físico/digital y el sistema inteligente pueden conjugarse para modificar internamente el espacio en búsqueda de un mayor confort del individuo sin necesidad de un agente completamente "externo"; se recurre aquí a la acción de los sistemas inteligentes embebidos en la arquitectura interna, otorgándole características de un "ambiente inteligente" con mayor capacidad de adaptación. Cuando a esta relación se le proporciona, además, la capacidad de detectar, interpretar y actuar con respecto a las emociones de los ocupantes, dicha relación se convierte en bidireccional generando una retroalimentación constante entre los tres elementos, donde el espacio se convierte en el nexo entre la tecnología y el ocupante. Así, el mencionado ocupante expresará su nivel de confort a través de sus emociones y la tecnología servirá como un mecanismo de detección que otorgará características de adaptabilidad al medio físico. Finalmente, se propone un modelo que grafica la relación que surge entre estas tres variables, las cuales podrán ser definidas por indicadores que representen el nivel tecnológico, nivel de adaptabilidad y nivel de confort. 
Es importante reconocer que el nuevo modelo de relación hombre-espacio propuesto es conceptual y requiere ser estudiado a mayor profundidad con casos de estudios que permitan contrastarlos con la realidad. Debido a que el tema aún es nuevo, hay poca disponibilidad de datos que permitan validar el modelo de manera cuantitativa y cualitativa.

Estableciendo la primera triada de variables que afectan los elementos de la domótica emocional, es importante estudiar su interrelación e influencia sobre el modelo propuesto. Ello incluye comprender el recorrido que realiza el flujo de información, que va desde la psique del ocupante hasta un modelo matemático de estimación del comportamiento humano.

Asimismo, el nivel de adaptabilidad de un espacio trae grandes retos a futuro. La pregunta es la siguiente: ¿hasta qué punto podemos automatizar y modular un espacio? Ello incluye reconocer cuáles son los parámetros físicos que pueden ser manejados para influir sobre los usuarios y cómo se integran al entorno; luego, investigar el potencial de muebles u objetos adaptables al estado del ánimo.

En el caso del nivel de confort, se halló poca información que cuantifique o modele cómo los parámetros en un entorno afectan al ocupante. Ello hace pensar que existe un campo fértil para sistematizar la regulación de las emociones humanas a partir de parámetros físicos medibles en ambientes cerrados.

\section{REFERENCIAS}

Aiello, M., Marchese, M., Busetta, P., y Calabrese, G. (2004). Opening the Home: A Web service approach to domotics. Trento: University of Trento.

Al Horr, Y., Arif, M., Katafygiotou, M., Mazroei, A., Kaushik, A., y Elsarrag, E. (2016). Impact of indoor environmental quality on occupant well-being and comfort: A review of the literature. International Journal of Sustainable Built Environment, 5(1), 1-11.

Barsky, J., y Nash, L. (2002). Evoking Emotion: Affective Keys to Hotel Loyalty. The Cornell Hotel and Restaurant Administration Quarterly, 43(1), 39-46.

Bollnow, O. F., y D'Ors, V. (1969). Hombre y espacio. Barcelona: Labor.

Bujdei, C., y Moraru, S. A. (2011). Ensuring Comfort in Office Buildings: Designing a KNX Monitoring and Control System. En 2011 Seventh International Conference on Intelligent Environments (pp. 222-229).

De Carvalho Correia, A. C., De Miranda, L. C., y Hornung, H. (2013). Gesture-based interaction in domotic environments: State of the art and $\mathrm{HCl}$ framework inspired by the diversity. En IFIP Conference on Human-Computer Interaction (pp. 300-3017). 
Eisenhardt, K. M. (1989). Building theories from case study research. Academy of management review, 14(4), 532-550.

Ekman, Paul and Keltner, Dacher (1970). Universal facial expressions of emotion. California mental health research digest, 8(4), 151-158.

El Murabet, A., y Abtoy, A. (2019). Understanding the Ambient Assisted Living systems: concepts, architectural trends and challenges. International Journal of Open Information Technologies, 7(1).

Fernández-Caballero, A., Martínez-Rodrigo, A., Pastor, J. M., Castillo, J. C., LozanoMonasor, E., López, M. T., et al. (2016). Smart environment architecture for emotion detection and regulation. Journal of biomedical informatics, 64, 55-73.

Fich, L. B., Jönsson, P., Kirkegaard, P. H., Wallergård, M., Garde, A. H., y Hansen, A. (2014). Can architectural design alter the physiological reaction to psychosocial stress? A virtual TSST experiment. Physiology y behavior, 135, 91-97.

Fransson, N., Västfjäll, D., y Skoog, J. (2007). In search of the comfortable indoor environment: A comparison of the utility of objective and subjective indicators of indoor comfort. Building and Environment, 42(5), 1886-1890.

Hirsch, P. M., y Levin, D. Z. (1999). Umbrella advocates versus validity police: A lifecycle model. Organization Science, 10(2), 199-212.

Montalbana, I., Garzo, A., y León, E. (2009). Emotion-aware intelligent environments: A user perspective. Intelligent Environments, 421-428.

Navarro-Tuch, S. A., Bustamante-Bello, M. R., Ávila-Vázquez, R., Izquierdo-Reyes, J., Ramírez-Mendoza, R., Pablos-Hach, J. L. y Gutiérrez-Martínez, Y. (2016). Emotional domotics: Inhabitable space variable control for the emotions modulation. In Springer (Ed.), Proceedings of SAI Intelligent Systems Conference (1034-1042).

Navarro-Tuch, S. A., Bustamante-Bello, M. R., Molina, A., Izquierdo-Reyes, J., Ávila-Vázquez, R., Pablos-Hach, J. L., y Gutiérrez-Martínez, Y. (2018). Inhabitable space control for the creation of healthy interactive spaces through emotional domotics. International Journal on Interactive Design and Manufacturing (IJIDeM), 12(4), 1337-1347.

Russell, J. A. (1980). A circumplex model of affect. Journal of Personality and Social Psychology, 39(6), 1161-1178.

Ryu, K., y Jang, S. (2008). Retracted Article: Influence of restaurants' physical environments on emotion and behavioral intention. The Service Industries Journal, 28(8), 1151-1165.

Stanley Okwudili, A. (2015). The influence of architecture on human behavior. Epoch of Science. Recuperado de https://elibrary.ru/item.asp?id=25429527

Valencia,H.(2019).Domóticaemocional,elsistemaquetecambiaelánimo. Recuperadode http://cienciamx.com/index.php/ciencia/salud/15922-domotica-emocional -sistema-animo 